\title{
DESIGN AND MULTIPLIERLESS IMPLEMENTATION OF TWO-CHANNEL BIORTHOGONAL IIR FILTER BANKS WITH LOW SYSTEM DELAY
}

\author{
J. S. Mao*, W.-S. Lu*, S. C. Chan ${ }^{\dagger}$, and A. Antoniou* \\ ${ }^{*}$ Dept. of Electrical and Computer Engineering, \\ University of Victoria, Victoria, B.C., Canada V8W 3P6 \\ ${ }^{\dagger}$ Dept. of Electrical and Electronic Engineering, \\ University of Hong Kong, Pokfulam Road, Hong Kong
}

\begin{abstract}
An efficient method for the design of low-delay two-channel, perfect reconstruction IIR filter banks is proposed. The design problem is formulated in terms of minimax designs of a general stable IIR filter that can be obtained using semidefinite programming and an FIR filter that can be obtained using the Remez exchange algorithm. A multiplierless implementation on this filter bank is also proposed and investigated.
\end{abstract}

\section{INTRODUCTION}

Low system delay is often desired for filter banks used in subband coding, subband adaptive filtering, and many other applications [1-3]. An interesting problem formulation that can be used for the design of low-delay filter banks was proposed by Kim and Ansari in [4] and developed further by Phoong, Kim, Vaidyanathan, and Ansari in [5]. This formulation entails two independent functions of $z, a(z)$ and $\beta(z)$, that can be chosen to satisfy specified frequency-domain requirements while maintaining the perfect reconstruction property (PR). More recent work on this type of filter bank includes the method in [6] where $\alpha(z)$ and $B(z)$ are rational functions of $z$ that represent IIR filters and the method in [7] where both $\alpha(z)$ and $\beta(z)$ are polynomials in $z^{-1}$ that represent nonlinear-phase FIR filters. In this paper, we use the formulation in [4][5] to design low-delay filter banks but chose $\alpha(z)$ to be a polynomial in $z^{-1}$ that represents a linear-phase FIR filter and $\beta(\hat{z})$ to be a rational function of $z$ that represents an IIR filter. Thus $\alpha(z)$ can be obtained by designing the FIR filter using the Remez exchange algorithm whereas $\beta(z)$ can be obtained by designing the corresponding IIR filter by using semidefinite programming (SDP). Computer simulations demonstrate that the proposed method yields satisfactory designs in terms of transition width, stopband attenuation, and implementation complexity compared with several existing methods. A multiplierless implementation of such IIR filter bank is also proposed.

\section{DESIGN OF IIR ANALYSIS FILTER BANK}

\subsection{Lowpass Analysis Filter}

Consider the 2-channel biorthogonal maximally decimated filter bank shown in Fig. I where the analysis lowpass filter $\mathbf{H}_{0}$ and highpass filter $\mathbf{H}_{1}$ are characterized by

$$
H_{0}(z)=\frac{1}{2}\left[z^{-2 N}+z^{-1} \beta\left(z^{2}\right)\right]
$$

and

$$
H_{1}(z)=-\alpha\left(\hat{z}^{2}\right) H_{0}(z)+z^{-2 M-1}
$$

respectively, function $\alpha(z)$ is a polynomial in $z^{-1}$ and $\beta(z)$ is a rational function in $z$. The transfer functions of the synthesis filters are given by $G_{0}(z)=-H_{1}(-z)$ and $G_{1}(z)=H_{0}(-z)$. A filter bank of this type is known to have the perfect reconstruction (PR) property regardless of the choices of $\alpha(z)$ and $\beta(z)$ [4][5]. The formula of $H_{0}(z)$ in (1a) suggests that if we design a lowpass filter represented by $\beta(z)$ with passband $\left[0,2 \omega_{p}\right]$ and passband group delay $N-\frac{1}{2}$, then $\mathbf{H}_{0}$ will be a lowpass filter with passband $\left[0, \omega_{p}\right]$ and passband group delay $2 N[5]$.

Let us assume that $\beta(z)$ is the transfer function of an IIR filter of the form

$$
\beta(z)=\frac{b(z)}{z^{n-r} d(z)}=\frac{\sum_{i=0}^{n} b_{i} z^{n-i}}{z^{n-r} \sum_{i=0}^{r} d_{i} z^{r-i}}
$$

By formulating an error function and minimizing it using SDP, $\beta(z)$ can be determined. Assuming that the sampling frequency is $2 \pi$, an error function can be defined as

$$
E_{0}(\omega)=\beta\left(e^{j \omega^{\prime}}\right)-\beta_{d}(\omega)
$$

where $\beta_{d}(\omega)$ is the desired frequency response of the IIR filter and is equal to $e^{-j \omega(N-1 / 2)}$ for $\omega \in\left[0,2 \omega_{p}\right]$ and zero elsewhere. An approximation for $3(z)$ can be obtained by solving the minimization problem

$$
\begin{gathered}
\operatorname{minimize} \delta \\
\text { subject to: } \frac{W_{3}^{-2}(\omega)}{\left|d_{k-1}(\omega)\right|^{2}}\left|b(\omega)-d(\omega) \beta_{d}(\omega)\right|^{2} \leq \delta \\
\quad \text { for } \omega \in\left[0,2 \omega_{p}\right] \\
d(z) \neq 0 \text { for }|\hat{\imath}| \geq 1
\end{gathered}
$$

where $\delta=\max \left|E_{\mathbf{0}}(\omega)\right|, d_{k-1}(z)$ denotes the denominator polynomial $d(z)$ obtained in the $(k-1)$ th iteration, $\Pi_{3}^{-}(\omega) \geq 0$ is a weighting function, and the stability of the IIR filter is assured by the constraint in ( $3 \mathbf{c})$. Now if $\mathbf{x}=[\delta \mathbf{b} \mathbf{d}]^{T}$ with $\mathbf{b}=\left[b_{0} \cdots b_{n}\right]$ and $\mathbf{d}=\left[d_{0} \cdots d_{n}\right]$, then the constraint in (3b) is equivalent to

$$
\boldsymbol{\Gamma}=\left[\begin{array}{ccc}
\delta & a_{1} & a_{2} \\
a_{1} & 1 & 0 \\
a_{2} & 0 & 1
\end{array}\right] \succeq \mathbf{0} \text { for } \omega \in\left[0,2 \omega_{p}\right\}
$$


where the notation $\boldsymbol{\Gamma}(\mathbf{x}, \omega) \succeq \mathbf{0}$ denotes that matrix $\boldsymbol{\Gamma}(\mathbf{x}, \omega)$ is positive definite and $a_{i}$ for $i=1,2$ are known functions of $\mathrm{x}$ and $\omega[8]$. Using the well-known Lyapunov stability theory [9], it can be shown that $d_{k-1}(z)$ has all its zeros in the unit circle if and only if there exists a positive definite matrix $\mathbf{P}_{k-1}$ such that

$$
\left[\begin{array}{ll}
\mathbf{P}_{k-1}^{-1} & \mathbf{D}_{k-1} \\
\mathbf{D}_{k-1}^{-1} & \mathbf{P}_{k-1}
\end{array}\right] \succ \mathbf{0}
$$

where $\mathbf{D}_{k-1}$ is the canonical matrix obtained by using the coefficients of $d_{k-1}$ [8][9], and the notation $\succ \mathbf{0}$ denotes that the above matrix is positive definite. After the $(k-1)$ th iteration is complete, one can solve the Lyapunov equation $\mathbf{P}_{k-1}-\mathbf{D}_{k-1}^{T} \mathbf{P}_{k-1} \mathbf{D}_{k-1}=$ I for the positive definite solution of $\mathbf{P}_{k-1}$ and use it to construct the linear matrix inequality (LMI)

$$
\mathbf{Q}_{k}(\mathbf{x})=\left[\begin{array}{cc}
\mathbf{P}_{k-1}^{-1}-\tau \mathbf{I} & \mathbf{D} \\
\mathbf{D}^{T} & \mathbf{P}_{k-1}-\tau \mathbf{I}
\end{array}\right] \succeq \mathbf{0}
$$

in the $k$ th iteration to assure the stability of the IIR filter. In the above equation, a parameter $\tau>0$ is introduced to control the stability margin of the filter. The $k$ th iteration of the design can now be formulated as the SDP problem

$$
\begin{aligned}
& \text { minimize } \mathbf{c}^{T} \mathrm{x} \\
& \text { subject to: }\left[\begin{array}{cc}
\boldsymbol{\Gamma}(\mathbf{x}) & \mathbf{0} \\
\mathbf{0} & \mathbf{Q}_{k}(\mathbf{x})
\end{array}\right] \succeq \mathbf{0}
\end{aligned}
$$

where $\mathbf{c}=\left[\begin{array}{llll}1 & 0 & \cdots & 0\end{array}\right]^{T}$ and $\boldsymbol{\Gamma}(\mathbf{x})=\operatorname{diag}\left[\boldsymbol{\Gamma}\left(\mathbf{x}, \omega_{0}\right), \cdots, \boldsymbol{\Gamma}\left(\mathbf{x}, \omega_{m}\right)\right]$ with $\omega_{i}, 1 \leq i \leq m$ being a set of frequency points in the interval $\left[0,2 \omega_{p}\right]$. The optimization problem in (5) can be efficiently solved using the LMI toolbox of MATLAB. With $\beta(\hat{*})$ known, the analysis lowpass filter $\mathbf{H}_{0}$ can be obtained.

\subsection{Highpass Analysis Filter}

Now let us examine the design of the analysis highpass filter $\mathbf{H}_{1}$. If function $\alpha(z)$ is assumed to be the transfer function of a linearphase FIR filter of even length of the form $\alpha(z)=\sum_{i=0}^{N_{a}-1} \alpha_{i} \tilde{i}^{-1}$. we can define the error function for the analysis highpass filter $\mathbf{H}_{1}$ as

$$
\begin{aligned}
E_{1}(\omega) & =H_{1}\left(e^{j \omega}\right)-H_{1 d}(\omega) \\
& =-\alpha\left(e^{2 j \omega}\right) H_{0}\left(e^{j \omega}\right)+e^{-j(2 M+1) \omega}-H_{1 d}(\omega)
\end{aligned}
$$

where $H_{1 d}(\omega)$ is the desired frequency response of $\mathbf{H}_{1}$ and is equal to $e^{-j \omega(2 M+1)}$ for $\omega \in\left[\omega_{s}, \pi\right]$ and zero elsewhere. Now let $H_{0}\left(e^{j \omega}\right)=A(\omega) e^{-j 2 N \omega}$, where $A(\omega)$ is approximately equal to one in magnitude for $\omega \in\left[0, \omega_{p}\right]$, and let the frequency response of the FIR filter be $\alpha\left(e^{j \omega}\right)=e^{-j \omega\left(N_{\mathrm{a}}-1\right) / 2} \cos (\omega / 2) P_{\mathrm{a}}(\cos \omega)$, where $P_{\alpha}(\cos \omega)$ is a Chebyshev polynomial of order $N_{a} / 2-1$ that contains the coefficients of $\alpha(z)$. The error function in (6) can then be expressed as

$$
\begin{array}{r}
E_{1}(\omega)=e^{-j(2 M+1) \omega}\left[1-\cos (\omega) P_{\alpha}(\cos (2 \omega)) A(\omega)\right] \\
\text { for } \omega \in[0, \pi / 2]
\end{array}
$$

By setting $\partial\left|E_{1}(\omega)\right|^{2} / \partial P_{\alpha}$ to zero, we obtain the Chebyshev polynomial $P_{a}(\cos (2 \omega))$ that minimizes $\left|E_{1}(\omega)\right|^{2}$ as

$$
P_{\alpha}(\cos (2 \omega))=\frac{\Re[A(\omega)]}{|A(\omega)|^{2} \cos \omega} \text { for } \omega \neq \pi / 2
$$

Hence the design problem at hand can be formulated as the least pth minimization problem [10]

$$
\operatorname{minimize} \int_{I_{x}} W_{a}(x)\left|P_{a}(x)-P_{d}(x)\right|^{P} d x
$$

where $x=\cos (2 \omega), x_{s}=\cos \left(2 \omega_{p}\right), I_{x}$ is the interval defined by $I_{x}=\left(-1,-x_{s}\right) \cup\left(x_{s}, 1\right)$, and $W_{a}(\omega)$ is a weighting function given by $W_{\alpha}(x)=\cos (0.5 \arccos x)|A(0.5 \arccos x)|^{2}$. The optimization problem in (8) with $P=\infty$ can be solved using the Remez exchange algorithm [11]. With $\alpha(z)$ known. the analysis highpass filter $\mathbf{H}_{1}$ can be obtained.

\subsection{Selection of Design Parameters}

We now describe some simple guidelines for the selection of parameters $N, n, M$, and $N_{\sigma}$ for given design specifications. If filter $\mathbf{H}_{0}$ were a linear-phase balf-band FIR filter. its length could be predicted by using a formula due to Herrmann et al. [11] as

$$
L=\left\lfloor\frac{D-F B^{2}}{B}+1.5\right\rfloor
$$

where $B=\frac{\left|\omega_{s}-w_{p}\right|}{2 \pi}, F=11.012$,

$$
\begin{aligned}
D= & {\left[0.005309\left(\log \delta_{s_{0}}\right)^{2}+0.07114 \log \delta_{s_{0}}-0.4761\right] \log \delta_{s_{0}} } \\
& -\left[0.00266\left(\log \delta_{s_{0}}\right)^{2}+0.5941\left(\log \delta_{s_{0}}\right)+0.4278\right]
\end{aligned}
$$

In the above formulas, $\delta_{s_{0}}$ is the stopband ripple, $\omega_{p}$ and $\omega_{s}$ are the passband and stopband edges of the analysis lowpass filter, respectively; $\lfloor Q\rfloor$ denotes the largest integer smaller than $\mathrm{Q}$. Now if $\mathbf{H}_{0}$ is an IIR filter, then extensive simulations have shown that parameter $N$ could be taken to be one eighth of $L[10]$. i.e.,

$$
N=\left\lceil\frac{L}{8}\right\rceil
$$

where $\lceil Q\rceil$ denotes the smallest integer larger than $Q$. A value of $N$ smaller than this may still work, but the filter bank may exhibit undesirable artifacts in the transition band. Having chosen parameter $N$, the value $n=N+2$ usually leads to satisfactory designs. On the other hand, the values $M=3 N-1$ and $N_{a}=4 N$ lead to a highpass analysis filter $\mathbf{H}_{3}$. whose frequency performance is comparable with that of the lowpass analysis filter $\mathbf{H}_{0}$. In doing so, a good overall performance of the filter bank can be obtained.

\section{A CASE STUDY}

\subsection{Design of a Filter Bank with Continuous Coefficients}

We have applied the design method proposed in Section 2 to design an IIR filter bank with the following specifications: $\delta_{s_{0}}=$ $1.778 e-3, \omega_{p}=0.45 \pi$, and $\omega_{s}=0.55 \pi$. The length of a linearphase half-band FIR filter was predicted to be $L=60$; therefore, parameter $N$ for the low-delay IIR filter bank was chosen as $N=\lceil L / 8\rceil=8$; with $N$ known. the other parameters can be deduced as follows: $n=10, M=23$, and $N_{a}=32$. We first designed an FIR nonlinear-phase transfer function of order 35 , represented by $\beta_{l}(\approx)$, by using the method proposed in [7]. A balanced approximation method [13] was applied to the FIR transfer function $\beta_{l}(z)$ to yield a stable rational function $\beta_{0}(z)$ of order 10 . This $\beta_{0}(z)$ was then used as the initial guess in the SDP optimization described in Section 2. The SDP algorithm took 
one iteration to converge and the result was a stable rational function $\beta(z)$ that gave an IIR filter $\mathbf{H}_{0}$ with equiripple magnitude response. Next, the Remez exchange algorithm was applied to obtain a linear-phase FIR transfer function $\alpha(z)$ of order 31. The transfer functions $H_{0}(z), H_{1}(z), G_{0}(z)$, and $G_{1}(z)$ were then constructed using (1). The system delay of the filter bank was $2 M+2 N+1=63 \mathrm{~s}$. The performance of the filter bank designed was evaluated and compared with that of the designs obtained with several existing methods. These include the methods in [4][7] where the same filter bank formulation was applied but with different selections of $\beta(z)$ and $\alpha(z)$. Based on the method proposed in [4], we obtained an allpass transfer function $\beta(z)$ of order 8 and a linear-phase FIR transfer function $\alpha(z)$ of order 31 . By using the method proposed in [7], we obtained a nonlinearphase FIR transfer function $\beta(\xi)$ of order 35 and a linear-phase FIR transfer function $\alpha(z)$ of order 31 . The linear-phase FIR filter bank with 32 lattice stages proposed in [14] was also considered in our comparison study. All the designs have the same system delay of $63 \mathrm{~s}$.

The frequency responses of $\alpha(z), \beta(z)$, and analysis IIR filters $\mathbf{H}_{0}$ and $\mathbf{H}_{1}$ are shown in Figs. 2(a)-(c). The performance of the designs is illustrated in Table I, where $\Delta \omega=\omega_{p}-\omega_{s}$ is the width of transition band for both $\mathbf{H}_{0}$ and $\mathbf{H}_{1}$, and $\delta_{s_{0}}, \delta_{s_{1}}$ are the stopband attenuations of filters $\mathbf{H}_{0}$ and $\mathbf{H}_{1}$, respectively. It is observed that the IIR filter bank designed by the proposed method outperforms the other methods in that it yields a narrower transition width and improved stopband attenuation. With regard to the implementation complexity, the allpass function of order 8 is implemented with 8 multipliers and 24 adders [2], the IIR function $\beta(z)$ is realized in terms of a 10 -stage lattice and ladder structure [11], and the linear-phase FIR $\alpha(z)$ is implemented using the direct form [12]. It is noted that the proposed method requires less multiplications and additions (at the original sampling rate) compared to the method in [7] but is not as economical as the methods in [4] and [14].

\subsection{Multiplierless Implementation of the Filter Bank}

It is advantageous to implement a filter bank without multiplications. A commonly used approach is to employ individual filters with coefficients that can be expressed as sums of a small number of power-of-two terms. In this way the filters can be implemented in terms of additions of shifted versions of the input. In what follows, we call such filter banks as filter banks with sum-of-powerof-two (SP2) coefficients. Optimal implementation of filter banks with SP2 coefficients can be achieved by using techniques such as simulated annealing or genetic algorithms but these methods require a large amount of computation. The approach we take here can be described as follows. First we design a filter bank with continuous coefficients. Next we assign a certain number of bits for each coefficient based on its magnitude, and determine its lower and upper SP2 bounds. For the filter bank designed in Section 3.1 there were 21 coefficients in $3(z)$, five of which were very small in magnitude and were not sensitive to quantization; these were fixed to their nearest SP2 values. Then through an exhaustive search we selected either the upper bound or the lower bound for each of the 16 remaining coefficients such that the objective function

$$
\begin{array}{r}
E_{k}\left(H_{k}\right)=\max \left\|H_{q k}(\omega)-H_{k}(\omega)\right\|, \\
k:=0,1, \omega \in[0,2 \pi]
\end{array}
$$

is minimized. In (11) $H_{q k}(\omega)$ for $k=0,1$ are the frequency responses with the coefficients quantized and $H_{k}(\omega)$ for $k=0,1$ are the corresponding desired frequency responses. For the linearphase FIR transfer function $a(z)$, there were 16 coefficients that needed to be quantized to their SP2 values, and the exhaustive search was also performed as for transfer function $\beta(z)$. The search for the optimal SP2 coefficients for $\beta(z)$ and $\alpha(z)$, which involved the selection of the optimal combination out of $2^{16} \mathrm{com}-$ binations in each case, was carried out on a Pentium II $400 \mathrm{MHz}$ PC. The resulting magnitude responses of the multiplierless 2channel IIR filter bank are depicted in Fig. 2(d). The average number of power-of-two terms used was 2.9. It is observed that the filter bank with SP2 coefficients still offers more than $50 \mathrm{~dB}$ stopband attenuation.

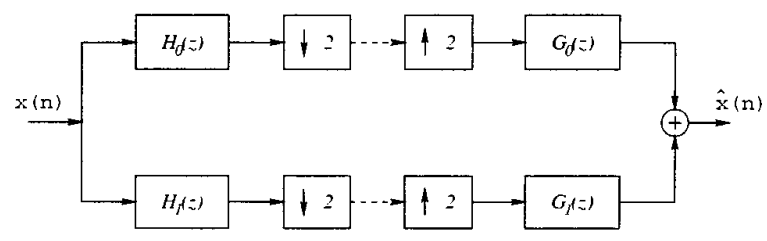

Figure 1: A two-channel maximally decimated filter bank.

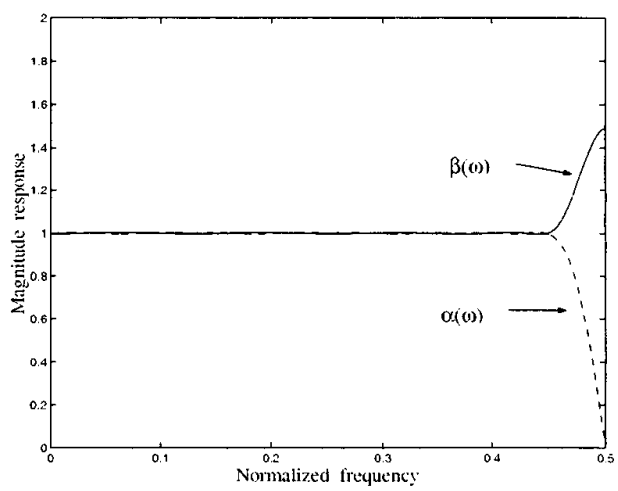

(a)

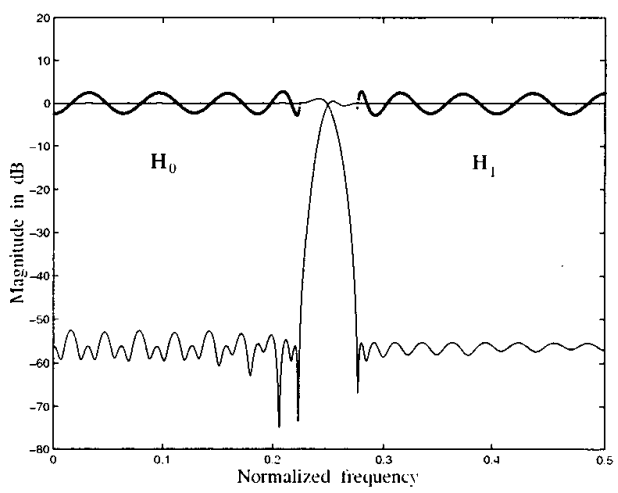

(b) 

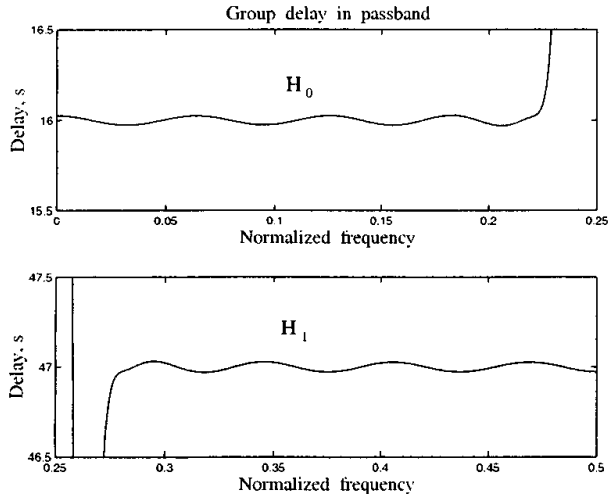

(c)

Table 1: Performance Comparisons

\begin{tabular}{|c|c|c|c|c|}
\hline & Allpass [4] & Non-LP FIR [7] & LP FIR FB [14] & Proposed IIR \\
\hline$\triangle \omega^{\prime}$ & 0.1 & 0.1 & 0.172 & 0.1 \\
$\delta_{s_{0}}(\mathrm{~dB})$ & 45.5 & 55.2 & 42.5 & 55.5 \\
$\delta_{s_{1}}(\mathrm{~dB})$ & +5 & 52.7 & 42.5 & 52.8 \\
Mult. & 12 & 26 & 17 & 23.5 \\
Add. & 28.5 & 34 & 49 & 31.5 \\
\hline
\end{tabular}

\section{ACKNOWLEDGMENT}

The authors are grateful to PMC-Sierra, Micronet, NCE Program, and the Natural Sciences and Engineering Research Council of Canada for supporting this work.

\section{REFERENCES}

[1] T. A. Ramstad, "IIR filter banks for subband coding of images," Proc. IEEE ISCAS'88, vol. 1, pp. 827-830, June 1988.

[2] P. P. Vaidyanathan, Multirate Systems and Filter Banks, Englewood Cliffs, NJ: Prentice Hall. 1992.

[3] P. A. Naylor, Q. Tanrikulu and A. G. Constantinides, "Subband adaptive filtering for acoustic echo control using allpass polyphase IIR filterbanks," IEEE Trans. Speech and audio Processing, vol. 6, no. 2, pp. 143-155, March 1998.

[4] C. W. Kim and R. Ansari, "IIR/IIR exact reconstruction filter bank with applications to subband coding of images." Proc. 34th Midwest CAS Symp., vol. 1, pp. 227-230, May 1991.

[5] S. M. Phoong. C. W. Kim. P. P. Vaidyanathan, and R. Ansari, "A new class of two-channel biorthogonal filter banks and wavelet bases," IEEE Trans. Signal Processing, vol.43, no. 3. pp. 649-664, March 1995.

[6] X. Zhang and T. Yoshikawa, "Design of two-channel stable IIR perfect reconstruction filter banks." IEICE Trans. Fundamentals, vol. E81-A. no.8, pp. 1592-1597, August 1998.

[7] J. S. Mao, S. C. Chan, and K. L. Ho, "Design of two-channel PR FIR filter banks with low system delay," Proc. IEEE IS$C A S^{\prime 2} 2000$, vol. I, pp. 627-630, May 2000.

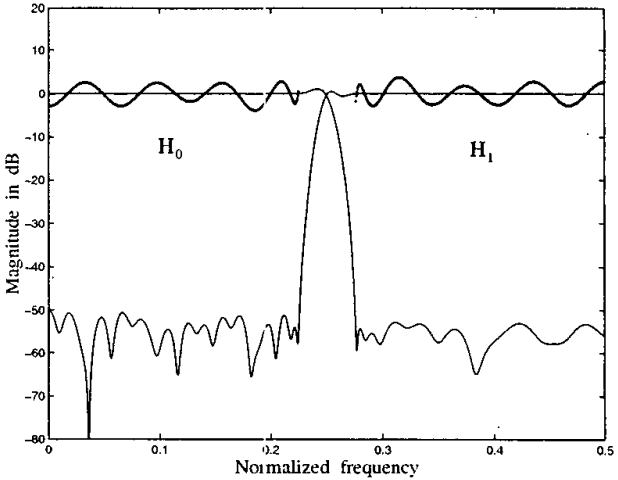

(d)

Figure 2: Two-channel low-delay IIR filter bank. (a) Magnitude responses of IIR filters represented by $\beta(z)$ (solid line) and the linear-phase FIR filter represented by $\alpha(z)$ (dashed line); (b) magnitude responses of the analysis IIR filters; (c) passband group delays of the analysis IIR filters; (d) magnitude responses of multiplierless IIR analysis filter bank. (The heavy solid lines in (b) and (d) are the passband gains of filters $\mathbf{H}_{0}$ and $\mathbf{H}_{1}$, multiplied by a factor of 200 in order to illustate the passband ripple.)

[8] W.-S. Lu, "Design of stable minimax IIR digital filters using semidefinite programming," Proc. IEEE ISCAS'2000, vol. I, pp. 355-358, May 2000.

[9] T. Kailath, Linear Systems, Englewood Cliffs, NJ: PrenticeHall, 1981.

[10] J. S. Mao, New Design and Factorization Methods for Perfection Reconstruction Filter Banks, Ph.D. Dissertation, The University of Hong Kong, April 2000.

[11] A. Antoniou, Digital Filiers: Analysis, Design, and Applications, 2nd ed., New York: McGraw-Hill, 1993.

[12] A. V. Oppenheim, R. W. Schafer and J. R. Buck, DiscreteTime Signal Processing, Upper Saddle River, NJ: PrenticeHall, 1999.

[13] B. C. Moore, "Principal component analysis in linear system: Controllability, observability, and model reduction," IEEE Trans. Automatic Control, vol. AC-26, pp. 17-31, 1981.

[14] T. Q. Nguyen and P. P. Vaidyanathan, "Two-channel perfectreconstruction FIR QMFi structures which yield linear phase analysis and synthesis filters," IEEE Trans. ASSP., vol. ASSP-37, pp. 676-690, May 1989. 